\title{
A Rare Case Report of Molar Pregnancy with Coexisting Fetus
}

\author{
${ }^{1}$ Richa S Nathani, ${ }^{2}$ Zeba Nisar
}

\section{ABSTRACT}

Aim: Here we report a molar pregnancy with a coexisting fetus progressing to a viable, healthy infant which is an extreme rarity, with the incidence of $0.005-0.01 \%$ of all the pregnancies.

Background: Partial mole is defined as excessive trophoblastic proliferation with normal and hydropic villi and presence of a fetus or a fetal circulation with nucleated cells. Partial molar pregnancy with a singleton live fetes is a rare condition. it is commonly seen with twin gestation and it is rare with singleton pregnancy

Case report: A 30 years old primigravida, referred from a private hospital at 38 weeks of gestation delivered a phenotypically normal and healthy infant $2200 \mathrm{gm}$ in weight by caesarean section. The placenta weighed $500 \mathrm{gms}$ and on sectioning revealed widespread, grape-like vesicles. Light microscopy revealed normal villi coexisting with villi showing hydropic change and cistern formation and diffuse circumferential trophoblastic hyperplasia consistent with molar change. A diagnosis of partial molar pregnancy with normal fetus was made.

Conclusion: Very few twin pregnancies with a hydatidiform mole and a foetus continue to term as they often have spontaneous or induced terminations for maternal complications Management of molar changes associated with normal appearing fetus still remains challenging as not many studies have been done due to rarity of this disorder.

Clinical significance: Though the general trend is to terminate pregnancy in foetus with coexistent mole in anticipation of complications, under close surveillance, optimal outcomes can be achieved. Monitoring of serum $\beta$-hCG, serial ultrasound for fetal growth, size of molar component, and theca lutein cysts can help to predict good patient outcomes.

Keywords: Beta-hCG, Molar pregnancy.

How to cite this article: Nathani RS, Nisar Z. A Rare Case Report of Molar Pregnancy with Coexisting Fetus. Int J Recent Surg Med Sci 2018;4(1):37-39.

Source of support: Nil

Conflict of interest: None

\footnotetext{
${ }^{1}$ Senior Resident, ${ }^{2}$ Consultant Pathologist

${ }^{1}$ Blood Bank, Employees State Insurance Model Hospital Basaidarapur, New Delhi, India

${ }^{2}$ The Oncopathology Centre, Mahatma Gandhi Cancer Hospital Miraj, Maharashtra, India

Corresponding Author: Richa S Nathani, Senior Resident Blood Bank, Employees State Insurance Model Hospital Basaidarapur, New Delhi, India, e-mail: richasnathani@ gmail.com
}

\section{INTRODUCTION}

A partial molar pregnancy is described as variation of molar pregnancy in which an embryo either develops partially or with multiple structural anomalies. ${ }^{1}$ In such an abnormal pregnancy, the egg usually receives two sets of chromosomes from the father, because two sperm have fertilized the egg.

The egg thus has 69 chromosomes instead of the normal $46 .{ }^{1}$ Most pregnancies in which molar changes have been reported in association with a normal fetus represent a dizygotic twin pregnancy, with one complete hydatidiform mole and the other normal twin with clearly distinguishable molar regions in the placenta. ${ }^{2}$

Here we present a case of singleton pregnancy in which placental molar change was associated with normal appearing fetus.

\section{CASE REPORT}

A 30-year-old primigravida, referred to us from a private hospital at 38 weeks of gestation, delivered a phenotypically normal and healthy newborn 2,200 gm in weight by cesarean section. She was an unbooked case. The placenta weighed $500 \mathrm{gm}$ and on sectioning revealed widespread, grape-like vesicles (Figs 1 and 2).

On light microscopy, the sections studied revealed normal villi pattern with few coexisting villi showing hydropic change and cistern formation, and at places diffuse circumferential trophoblastic hyperplasia was

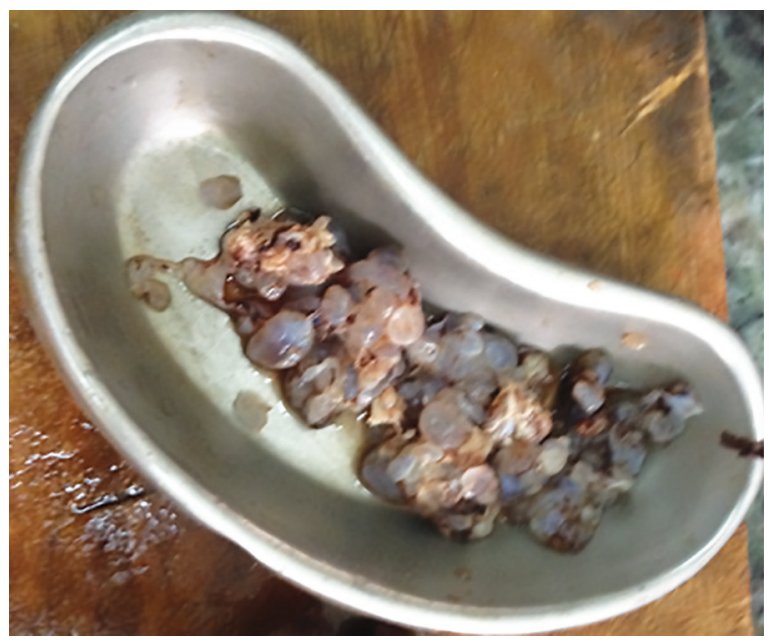

Fig. 1: Gross appearance of the hydatidiform mole with grape-like tissue 


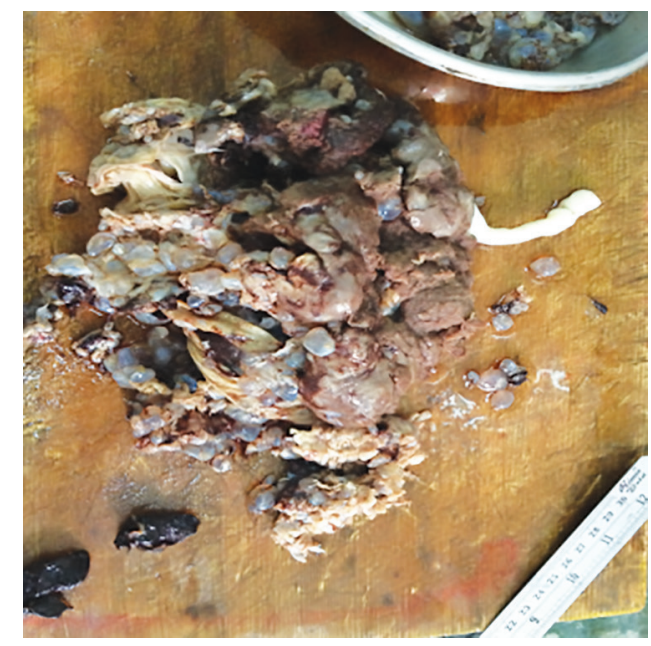

Fig. 2: Placenta with an attached umbilical cord and molar mass

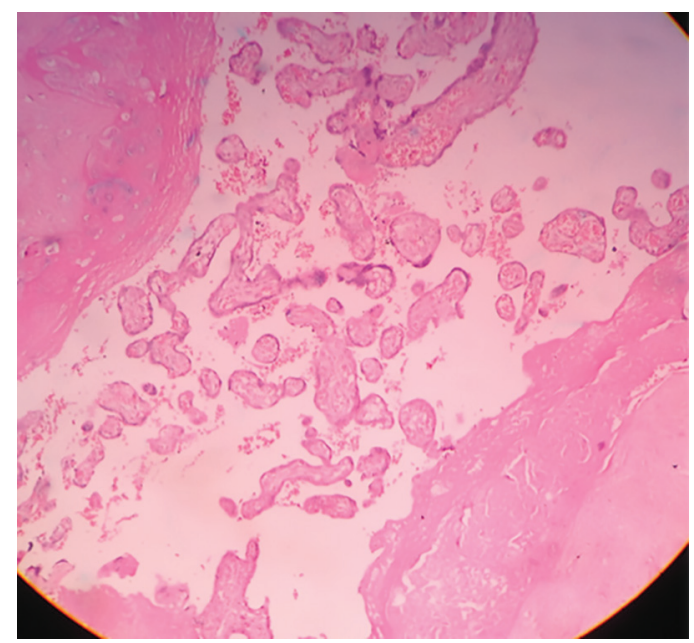

Fig. 4: Foci of chorangiosis: Multiple (>10) vascular profiles

evident, which was consistent with molar change (Fig. 3). Few areas of morphologically normal third trimester villi with syncytial knots and chorangiosis in some areas showing central cistern formation and occasional villi showed hyaline change (Fig. 4). A diagnosis of partial molar pregnancy with normal fetus was made.

\section{DISCUSSION}

Gestational trophoblastic disease constitutes a diverse group which comprises lesions that include abnormally formed placentas (hydatidiform moles), benign tumorlike lesions, and trophoblastic neoplasms ${ }^{3}$ (Table 1).

In partial molar pregnancy, there is usually an abnormal fetus with triploid genotype that is associated with a large placenta showing cystic changes.

The incidence of such normal diploid fetus associated with partial molar placenta is extremely rare. Here we report a case of partial molar pregnancy in which a normal appearing fetus was found to coexist. Partial molar pregnancy with coexisting fetus has an incidence

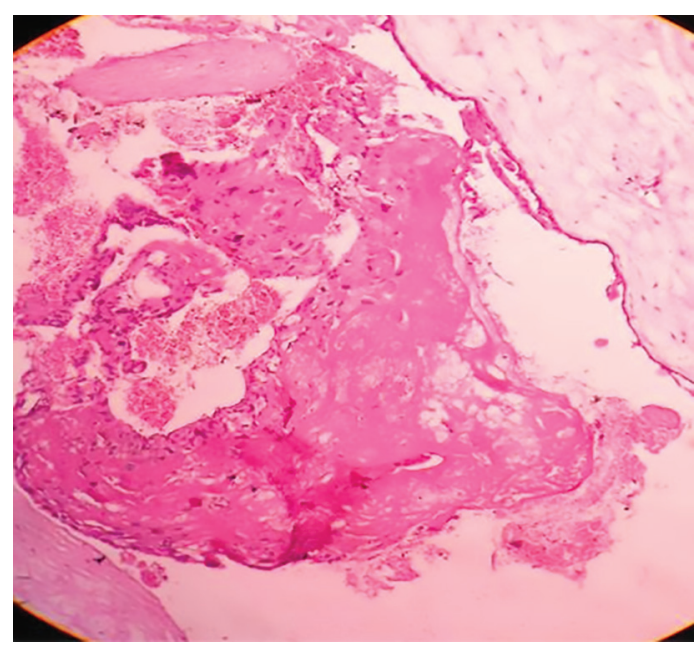

Fig. 3: Villi showing hydropic change and cistern formation

Table 1: New (4th edition) World Health Organization classification of gestational trophoblastic diseases

\begin{tabular}{l}
\hline Hydatidiform mole (abnormally formed placenta) \\
Complete mole \\
Partial mole \\
Invasive mole \\
Trophoblastic tumor (neoplastic disease) \\
Gestational choriocarcinoma \\
Placental site trophoblastic tumor \\
Epithelioid trophoblastic tumor \\
Tumor-like condition (benign lesion) \\
Exaggerated placental site reaction \\
Placental site nodule \\
\hline Adapted from Kurman et al ${ }^{4}$
\end{tabular}

of 0.005 to $0.01 \%$ of all the pregnancies. ${ }^{4}$ It usually is derived from the union of a haploid normal oocyte with two sperms, leading to dispermic fertilization and producing a triploid set of chromosomes. ${ }^{5}$

However, in some situations, molar changes in placenta maybe associated with a normal diploid fetus and in such cases other possibilities should be considered. The first is a twin pregnancy with one normal fetus (normal placenta) and another is a complete mole, ${ }^{6}$ so the first evaluation is looking for a normal separate placenta. In cases of a singleton normal fetus showing partial molar placenta, it is necessary to have normal karyotype for the fetus to survive in utero, although its placenta can have some chromosomal variation, from diploidy of the amnion to triploidy of the chorionic villi. ${ }^{7}$ Management of such molar changes associated with normal appearing fetus still remains a challenge.

The serum beta-human chorionic gonadotropin ( $\beta$-hCG) level is a helpful marker. When serum $\beta$-hCG level is greater than $106 \mathrm{mIU} / \mathrm{mL}$, termination of pregnancy should be considered. In contrast, in cases of successful pregnancy outcomes with viable fetuses, the serum 
$\beta$-hCG level usually starts to decline from the beginning of the second trimester, and sonography usually reveals a decrease in the size of the molar portion of the placenta. ${ }^{8}$

\section{CONCLUSION}

Pregnancy with hydatidiform molar changes and a coexisting live fetus requires a thorough evaluation, and pregnancy may be continued under close surveillance for an optimal outcome. Monitoring of serum $\beta$-hCG, serial ultrasound for fetal growth, size of molar component, and theca lutein cysts can help to predict good patient outcomes.

Management of molar changes associated with normal appearing fetus still remains challenging as not many studies have been done due to the rarity of this disorder.

\section{REFERENCES}

1. American Pregnancy Association. Molar pregnancy: symptoms, risks and treatment. [Online] Available at: http://americanpregnancy.org/pregnancy-complications/ molarpregnancy/
2. Hsieh CC, Hsieh TT, Hsueh C, Kuo DM, Lo LM, Hung TH. Delivery of a severely anaemic fetus after partial molar pregnancy: clinical and ultrasonographic findings. Hum Reprod 1999;14:1122-1126.

3. Kurman RJ et al. Gestational Trophoblastic Disease. Mills S.E. (ed) Sternberg's Diagnostic Surgical Pathology. 6th edition. Wolters Kluwer; 2015. pp. 2297-2319.

4. Suzuki M, Matsunobu A, Vakita K, Osanai K. Hydatidiform mole with surviving co-existent fetus. Ombt Gynecol 1980; 56:384-388.

5. Vaisbuch E, Ben-Arie A, Dgani R, Perlman S, Sokolovsky N, Hagay Z. Twin pregnancy consisting of a complete hydatidiform mole and co-existent fetus: report of two cases and review of literature. Gynecol Oncol 2005;98:19-23.

6. Changchien CC, Eng HL, Chen WJ. Twin pregnancy with hydatidiform mole $(46, \mathrm{XX})$ and a coexistent fetus $(46, \mathrm{XY})$ : report of a case. J Formos Med Assoc 1994;93:337-339.

7. Sarno AP, Moorman AJ, Kalousek DK. Partial pregnancy with fetal survival: an unusual example of confined placental mosaicism. Obstet Gynecol 1993;82:716-719.

8. Radhouane Achour, Imen Ben Aissa, Khaled Neji. Twin pregnancy with both complete hydatiform mole and coexistent alive fetus:case report. Asian Pac J Reprod 2015;4(4):331-333. 